PAPER

\title{
Inflammatory markers, rather than conventional risk factors, are different between carotid and MCA atherosclerosis
}

\author{
O Y Bang, P H Lee, S R Yoon, M A Lee, I S Joo, K Huh
}

See Editorial Commentary, p 1045

J Neurol Neurosurg Psychiatry 2005;76:1128-1134. doi: 10.1136/jnnp.2004.054403

See end of article for authors' affiliations

......................

Correspondence to: Dr Oh Young Bang Department of Neurology, College of Medicine, Ajou University, Woncheondong San 5, Paldal-ku, Suwon, Kyungki-do, 442749, Republic of Korea: nmboy@unitel.co.kr

Received

17 September 2004

Revised version received

8 December 2004

Accepted 7 January 2005
Background: The apparent differences in risk factors for intra- and extracranial atherosclerosis are unclear and the mechanisms that underlie strokes in patients with intracranial atherosclerosis are not well known. We investigated the conventional vascular risk factors as well as other factors in stroke patients with large artery atherosclerosis.

Methods: Using diffusion weighted imaging (DWI) and vascular and cardiologic studies, we selected patients with acute non-cardioembolic cerebral infarcts within the middle cerebral artery (MCA) territory. Patients were divided into two groups: those with atherosclerotic lesions on the carotid sinus $(n=112)$ and those with isolated lesions on the proximal MCA $(n=160)$. Clinical features, risk factors, and DWI patterns were compared between groups.

Results: There were no differences in conventional risk factors, but markers for inflammation were significantly higher in patients with carotid atherosclerosis than in those with isolated MCA atherosclerosis $(p<0.01$ for both). After adjustments for age/sex and the severity of stroke, an inverse correlation was observed between C-reactive protein levels and MCA atherosclerosis (odds ratio 0.57 per $1 \mathrm{mg} / \mathrm{dl}$ increase; $95 \%$ confidence interval 0.35 to $0.92 ; p=0.02$ ). Internal borderzone infarcts suggestive of haemodynamic causes were the most frequent DWI pattern in patients with MCA occlusion, whereas territorial infarcts suggesting plaque ruptures were most common in those with carotid occlusion.

Conclusions: Our results indicate that inflammatory markers, rather than conventional risk factors, reveal clinical and radiological differences between patients with carotid and MCA atherosclerosis. Plaques associated with MCA atherosclerosis may be more stable than those associated with carotid atherosclerosis.
$\mathrm{C}$ arotid and coronary atherosclerosis have many clinical and pathological features in common. However, the pathophysiological characteristics of intracranial atherosclerosis and the mechanisms that underlie strokes in patients with this condition are unknown for the following reasons. First, pathological specimens of intracranial atherosclerosis can be obtained only from autopsies; therefore, relatively few cases with pathological data on intracranial atherosclerosis have been reported. ${ }^{1-4}$ Second, unlike carotid atherosclerosis, sonographic evaluation of the morphology of plaques in intracranial vessels is severely limited. Third, the clinical and radiological features of intracranial atherosclerosis have not been clarified. Finally, the risk factors for intra- and extracranial atherosclerosis may differ, and these differences may account for the low frequency of carotid bifurcation disease in ethnic groups that are prone to develop intracranial diseases.

However, the apparent differences in risk factors for intraand extracranial arteries are unclear. Consequently, patients with intracranial stenosis receive the same treatment as those with carotid atherosclerosis. Intracranial atherosclerosis appears to cause $8-10 \%$ of all acute strokes in the United States, and in one Chinese study, more than half of patients with acute ischemic stroke had intracranial stenosis. ${ }^{5}$ Intracranial stenosis is probably just as significant as carotid bifurcation stenosis.

We hypothesised that the risk factors for stroke and the infarct patterns in patients with intracranial atherosclerosis differ from those associated with carotid atherosclerosis. Therefore, we compared clinical and radiological features, as well as conventional and novel risk factors for stroke, between patients with occlusive lesions on the carotid sinus and patients with occlusive lesions on the proximal portion of the middle cerebral artery (MCA).

\section{METHODS}

\section{Patient selection and grouping}

Between July 2000 and October 2003 we prospectively studied consecutive patients with acute symptomatic MCA infarcts. Such patients were identified as having (a) suffered focal symptoms that lasted for $>24 \mathrm{~h}$, (b) been observed within 7 days of the onset of symptoms, and (c) relevant lesions within the MCA territory as assessed using diffusion weighted imaging (DWI). All patients consented to participate in this study. We excluded patients with MCA infarcts associated with (a) potential sources of cardioembolism, (b) small arterial disease, (c) other etiologies (such as carotid dissection, hypercoagulable state, vasculitis, and complicated migraine), (d) incomplete workups, (e) undetermined causes of stroke, according to the previously published classification, ${ }^{6}$ and (f) isolated lesions on the intracranial portion of the internal carotid artery (ICA) (fig l).

Of 1088 patients admitted during the study period, 272 were included in the study. Of these, 150 (55\%) were men and $122(45 \%)$ were women. The average (SD) age of the study population was 62.4 (12.3) years (range: 37-87).

Based on an angiographic study, we divided patients who had acute symptomatic MCA territory infarcts into two groups, namely (a) those with occlusive lesions on the carotid

Abbreviations: CAOD, coronary artery occlusive disease; CRP, Creactive protein; DWI, diffusion weighted imaging; ICA, internal carotid artery; MCA, middle cerebral artery; MMP-9, matrix metalloproteinase9; NIHSS, National Institutes of Health Stroke Scale 


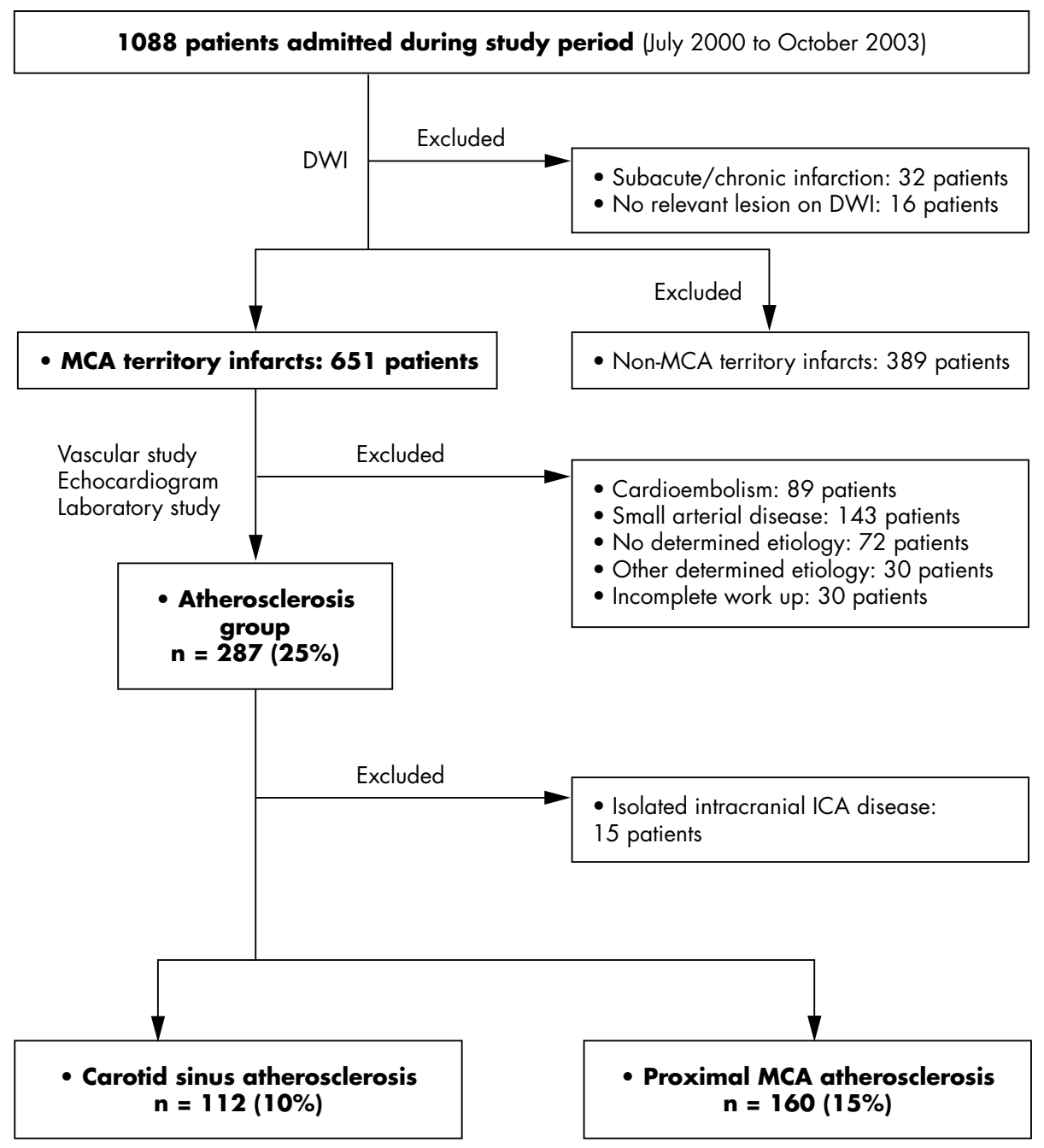

Figure 1 Patient selection and grouping.

sinus (carotid-type patients) and (b) those with occlusive lesions on the proximal portion of the MCA, but with no significant stenosis on the intra- or extracranial ICA (MCAtype patients); 112 and 160 patients were classified as carotid-type and MCA-type patients, respectively. Digital subtraction angiography was performed in 83 patients and magnetic resonance angiography in the remaining 189 patients, at 1.8 (SD 2.3) days after the onset of symptoms. An occlusive lesion was defined, according to the previously published methods, as $>50 \%$ stenosis or occlusion of the large intracranial vessels and the carotid sinus. $^{78}$ DWI patterns and angiographic features were interpreted by two neurologists who were blind to the patients' clinical presentation. Agreement between the two observers for the presence of an occlusive lesion on conventional or magnetic resonance angiography was $91 \%$.

Assessment of clinical courses and laboratory workup Patients were evaluated according to a protocol that included demographic data, medical history, vascular risk factors, and the National Institutes of Health Stroke Scale (NIHSS) score, as in our previous study. ${ }^{9}$ Laboratory tests were performed in all the patients, including routine blood tests, brain magnetic resonance imaging ( $1.5 \mathrm{~T})$, and vascular studies, echocardiography, and electrocardiography.

\section{Novel risk factors for atherosclerosis}

Peripheral blood samples were drawn from each patients at the time of admission (mean $(\mathrm{SD})=1.6$ (2.1) days after symptom onset). As novel risk factors for atherosclerosis, serum levels of C-reactive protein (CRP) and fibrinogen were evaluated in all but 15 patients. In addition, serum level and activity of matrix metalloproteinase-9 (MMP-9) were evaluated in selected patients who enrolled between March and October 2003. We excluded patients who were treated with thrombolysis or who had hypothermia which may influence the level of MMP-9. The plasma levels and activity of MMP-9 were measured with commercially available assay kits (Biotrak, Amersham, Little Chalfont, Bucks, UK). These determinations were performed by a researcher who was blind to the clinical and radiological data.

\section{DWI patterns}

DWI patterns were classified into two groups, namely (a) territorial infarcts that suggested a plaque rupture with subsequent macrothrombi, and (b) non-territorial infarcts. The latter group included four subgroups according to previously published methods, ${ }^{10}{ }^{11}$ namely (a) striatocapsular infarcts (either small or large), (b) internal borderzone infarcts that suggested haemodynamic infarcts, ${ }^{12}$ (c) multiple small superficial infarcts that suggested thrombo-embolism, 
and (d) cortical infarcts (either small territorial, involving one MCA sub-territory, or cortical borderzone infarcts). Based on the DWI data, small, multifocal ischemic lesions $<1 \mathrm{~cm}$ in diameter and involving the cortex or centrum ovale were classified as multiple small superficial infarcts. ${ }^{13}$ Multilocular chainlike lesions, confluent striated lesions, or solitary lesions that were located in the supraventricular or paraventricular region were defined as internal borderzone infarcts. ${ }^{14}$ Territorial infarcts were large infarcts that involved at least two of three MCA sub-territories, namely deep, superior, or inferior. ${ }^{11}$ We also calculated the volumes of all DWI lesions in each patient. All axial DWI lesion volumes were computed by multiplying the area on each slice by the section thickness (TR, $10000 \mathrm{~ms}$; TE, 104 ms; slice thickness $7 \mathrm{~mm}$; no gap).

\section{Statistical analysis}

Differences between the groups in the clinical and radiological features, and risk factors, were examined using $\chi^{2}$ or Fisher's exact tests and Student's t tests or one way ANOVA with post hoc analysis. An independent association of inflammatory markers (CRP and fibrinogen) with MCA atherosclerosis was tested; we performed a multiple logistic regression analysis to determine whether any inflammatory markers were independent predictors for MCA atherosclerosis, over and above the severity of the stroke (the NIHSS score at admission and the DWI lesion volume), the previous history of coronary artery occlusive disease (CAOD), or the age and sex of the patient. Those that were significant at the 0.2 level were entered into the initial multivariate model. When the most parsimonious model was obtained by backward stepwise elimination of the non-significant factors, each of the excluded variables was again entered separately into the model to test its contribution to the final model. Results are given as odds ratios (OR) estimates of relative risk, with $95 \%$ confidence interval (CI). Statistical significance was established at $\mathrm{p}<0.05$.

\section{RESULTS}

\section{General data}

Angiographic observations of relevant and irrelevant vessels are shown in table 1. Tandem occlusive lesions on the contralateral carotid artery were more prevalent in carotidtype than in MCA-type patients ( $14 v 4$ patients, respectively; $\mathrm{p}=0.002$ ), whereas intracranial stenosis on the contralateral side or basilar artery was more prevalent in the latter than in the former group ( $42 v 15$ patients, respectively; $\mathrm{p}=0.010$ ). Although total occlusion was more prevalent in carotid-type than in MCA-type patients (53 $v 50$ patients, respectively; $\mathrm{p}=0.007$ ), the degrees of stenosis were not different between the groups: $84.8 \%$ (SD 21.3\%) in carotid-type patients and $79.8 \%$ (SD 20.0\%) in MCA-type patients ( $\mathrm{p}>0.05$ ).

The clinical characteristics of the study group are presented in table 2. MCA-type patients were significantly younger than carotid-type patients $(\mathrm{p}=0.036)$. The NIHSS score at admission was significantly higher in carotid-type patients than in MCA-type patients, whereas the lacunar syndrome was more frequently observed in MCA-type patients. Clinical courses during admission were the same in each group. The use of aspirin and statin before the onset of symptom was not different between the groups ( $\mathrm{p}>0.05$ for both cases).

\section{Conventional and novel risk factors}

Despite the differences between the groups as regards the clinical features, the conventional risk factors were indistinguishable (table 2). A previous history of CAOD, such as myocardial infarction or angina, was observed more frequently in carotid-type patients than in MCA-type patients ( $16 \vee 7$ patients, respectively; $\mathrm{p}=0.004$ ).

The results of our investigation of novel risk factors for atherosclerosis are presented in tables 2 and 3. Markers for inflammation were significantly higher in carotid-type patients than in MCA-type patients $(p=0.007$ for CRP and $\mathrm{p}=0.005$ for fibrinogen). MMP-9 levels and activities were tested in 30 carotid-type patients and 52 MCA-type patients. MMP-9 levels and activities were also greater in the former group than in the latter group $(p=0.287$ for MMP-9 level and $\mathrm{p}=0.004$ for MMP-9 activity).

The DWI lesion volumes were different between the groups, being higher in the carotid-type patients than in the MCA-type patients (38.4 (SD 67.9) $\mathrm{ml}$ and 10.7 (SD 26.5 ) $\mathrm{ml}$, respectively; $\mathrm{p}=0.001$ ). To investigate the possible effects of the infarct itself on the aforementioned levels, linear correlation analysis between the novel risk factors and the DWI volume was performed (fig 2). The CRP and fibrinogen levels (which were higher in carotid-type patients than MCA-type patients, see above) were unrelated to the infarct volume $(\mathrm{r}=0.004, \mathrm{p}=0.566$ for CRP; $\mathrm{r}=0.066$, $\mathrm{p}=0.309$ for fibrinogen), whereas MMP-9 levels and activities were higher in patients with large infarct volumes $(\mathrm{r}=0.373, \mathrm{p}=0.001$ for MMP-9 level; $\mathrm{r}=0.483, \mathrm{p}<0.001$ for MMP-9 activity). A multiple logistic regression analysis was performed, and the results showed that the CRP level was independently associated with MCA atherosclerosis (OR: $0.569 ; 95 \%$ CI: 0.352 to $0.920 ; p=0.021)$ as was the initial DWI lesion volume (OR: 0.988 per $1 \mathrm{ml}$ increase; 95\% CI: 0.979 to $0.997 ; \mathrm{p}=0.009)$. Other clinical results, including epidemiologic data (age, $\mathrm{p}=0.358$; sex, $\mathrm{p}=0.264$ ), previous CAOD $(p=0.215)$, and the severity of neurologic deficits (the NIHSS score at admission, $\mathrm{p}=0.144$ ), were not associated with MCA atherosclerosis.

Besides the site of atherosclerosis, the levels of inflammatory markers were related to the presence of asymptomatic atherosclerosis and the degree of stenosis (table 3). Both CRP and fibrinogen levels were highest in carotid-type patients with contralateral carotid sinus or coronary atherosclerosis and patients with total occlusion of the carotid sinus that suggested a plaque rupture $(\mathrm{p}<0.01$ for all the cases $)$.

Table 1 Angiographic findings in the carotid and MCA group

\begin{tabular}{llc}
\hline & Carotid-type patients $(\mathbf{n}=112)$ & MCA-type patients $(\mathbf{n}=\mathbf{1 6 0})$ \\
\hline No combined lesion & 78 & 112 \\
Combined lesion on non-relevant site & 34 & 48 \\
Extracranial artery & $14(41 \%)$ & $4(8 \%)$ \\
Contralateral carotid & 14 & 4 \\
Intracranial artery & $15(44 \%)$ & $42(88 \%)$ \\
Contralateral MCA & 7 & 30 \\
Basilar artery & 4 & 8 \\
Contralateral MCA and basilar artery & 1 & 2 \\
Contralateral ICA siphon & 3 & $2(4 \%)$ \\
Combined intra- and extracranial artery & $5(15 \%)$ & \\
\hline ICA, internal carotid artery; MCA, middle cerebral artery. & & \\
\hline
\end{tabular}


Table 2 Clinical features and risk factors in patients of the carotid and MCA groups

\begin{tabular}{llll} 
& Carotid-type patients (n=112) & MCA-type patients (n= 160) & p \\
\hline Age, mean (SD) & $64.3(11.9)$ & $61.2(12.4)$ & 0.036 \\
Male sex & $68(61 \%)$ & $82(51 \%)$ & 0.663 \\
Clinical features & $12(11 \%)$ & $18(11 \%)$ & 0.840 \\
Preceding transient ischemic attack & $11(10 \%)$ & $5(3 \%)$ & 0.033 \\
Decreased consciousness at admission & $6.4(6.4)$ & $3.9(3.9)$ & 0.001 \\
NIHSS on admission, mean (SD) & $11(10 \%)$ & $41(26 \%)$ & 0.001 \\
Traditional lacunar syndrome & $65(58 \%)$ & $96(60 \%)$ & 0.746 \\
Conventional risk factors & $31(28 \%)$ & $56(35 \%)$ & 0.203 \\
Hypertension & $42(38 \%)$ & $56(35 \%)$ & 0.673 \\
Diabetes & $4.5(1.3)$ & $4.7(3.5)$ & 0.570 \\
Smoking & $112.6(34.5)$ & $119.2(38.7)$ & 0.160 \\
Serum cholesterol, mean (SD) & $29(26 \%)$ & $41(26 \%)$ & 0.960 \\
$\quad$ Ratio of total to high density lipoproteins & $16(15 \%)$ & $7(4 \%)$ & 0.004 \\
$\quad$ Low density lipoproteins (mg/dl) & $1.29(3.08)$ & $0.33(0.52)$ & 0.007 \\
Previous stroke history & $405.77(139.48)$ & $358.74(93.21)$ & 0.005 \\
Previous history of CAOD & $164.52(188.93)$ & $122.88(125.96)$ & 0.287 \\
Novel risk factors & $931.11(486.56)$ & $646.19(339.86)$ & 0.004 \\
CRP (mg/dl), mean (SD) & & \\
Fibrinogen (mg/dl), mean (SD) & & \\
MMP-9 level (ng/ml), mean (SD) & & \\
MMP-9 activity (ng/ml), mean (SD) & & & \\
\hline CAOD, coronary artery occlusive disease; MMP-9, matrix metalloproteinase-9. & & \\
\hline
\end{tabular}

\section{DWI lesion patterns}

According to the DWI data, territorial infarcts that involved two or more sub-territories were observed more frequently in carotid-type patients $(\mathrm{n}=23$ or $21 \%)$ than in MCA-type patients $(\mathrm{n}=7$ or $4 \%$; $\mathrm{p}<0.001$; fig 3$)$. In MCA-type patients, striatocapsular infarcts and internal borderzone infarcts were the most frequent type of DWI pattern in patients with MCA stenosis and occlusion, respectively. In the same manner, in carotid-type patients, multiple small superficial infarcts and territorial infarcts were the most frequent DWI pattern in patients with carotid stenosis and occlusion, respectively.

\section{DISCUSSION}

\section{MCA atherosclerosis is common in patients with non-} cardioembolic ischemic stroke

In the present study, MCA atherosclerosis was found to be more prevalent than carotid sinus atherosclerosis. Atherosclerotic involvement of the intracranial vessels occurs more frequently in Asians than in Caucasians. ${ }^{15}$ Consequently, the mechanisms that cause strokes and the treatment both of stroke victims and of patients with intracranial stenosis are particularly important for Asian individuals.

As shown in table 1, the pattern of vascular stenosis was striking in that atherosclerosis was clustered either in the extracranial or in intracranial arterial system. Our findings are similar to those of a previous study in which the absence of carotid bifurcation disease was a risk factor for the progression of intracranial atherosclerotic stenosis. ${ }^{16}$ The angiographic data in the present study suggest that there might be different risk factors for atherosclerosis that depend on whether patients have carotid- or MCA-type occlusion.

\section{Differences in risk factors for MCA and carotid atherosclerosis}

Nevertheless, the apparent differences in risk factors for intra- and extracranial arteries are unclear. Racial differences in the distribution of occlusive disease are probably due to racial differences in the prevalence of hypertension, diabetes, hypercholesterolaemia, and ischaemic heart disease. ${ }^{15}$ However, previous reports failed to document significant differences in the risk factor profiles among different ethnic groups. ${ }^{17}{ }^{18}$ In the present study, there were no significant differences in conventional risk factors between carotid- and MCA-type patients.

We investigated several novel substances that have emerged recently as risk factors for atherosclerosis. To our knowledge, levels of CRP, fibrinogen, and MMP-9 have not yet been compared between patients with carotid and MCA atherosclerosis. Recently, the Rotterdam study group reported that CRP levels predicted the progression of atherosclerosis that was measured at various sites in the arterial tree, but intracranial vessels were not evaluated. ${ }^{19}$ It is generally accepted that inflammation is important in plaque rupture, including an association between elevated CRP levels and an increased risk of ischemic events or high risk

Table 3 Levels of inflammatory markers

\begin{tabular}{|c|c|c|c|}
\hline Groups & No. of patients & CRP, mean (SD) & Fibrinogen, mean (SD) \\
\hline \multicolumn{4}{|l|}{ Presence of asymptomatic atherosclerosis } \\
\hline Isolated M1 lesion & 106 & $0.26(0.32)^{*}$ & $347.55(90.31) \ddagger$ \\
\hline M1 lesions with concomitant lesions on non-relevant vessels & 46 & $0.43(0.70)$ & $375.15(95.71)$ \\
\hline Isolated carotid sinus lesion & 74 & $1.25(3.0)$ & $402.23(141.14)$ \\
\hline $\begin{array}{l}\text { Carotid sinus lesions with contralateral carotid sinus or coronary } \\
\text { atherosclerosis }\end{array}$ & 31 & $1.44(3.29) \dagger$ & $417.38(136.60) \S$ \\
\hline \multicolumn{4}{|l|}{ Degree of symptomatic stenosis } \\
\hline Isolated MI stenosis & 109 & $0.31(0.37)$ & $355.89(91.58)^{* *}$ \\
\hline Isolated M1 occlusion & 43 & $0.39(0.79)$ & $366.69(98.42)^{* *}$ \\
\hline Carotid sinus stenosis & 57 & $0.87(2.24)$ & $391.78(115.71)$ \\
\hline Carotid sinus occlusion & 48 & $1.85(3.89)$ & 422.49 (163.39) t† \\
\hline
\end{tabular}

*Lower and thigher levels than other groups, on ANOVA, $p=0.006$; †lower and \$higher levels than other groups, on ANOVA, $p=0.008$; 9 higher levels than other groups, on ANOVA, $p=0.001 ;{ }^{* *}$ lower and tthigher levels than other groups, on ANOVA, $p=0.010$. 

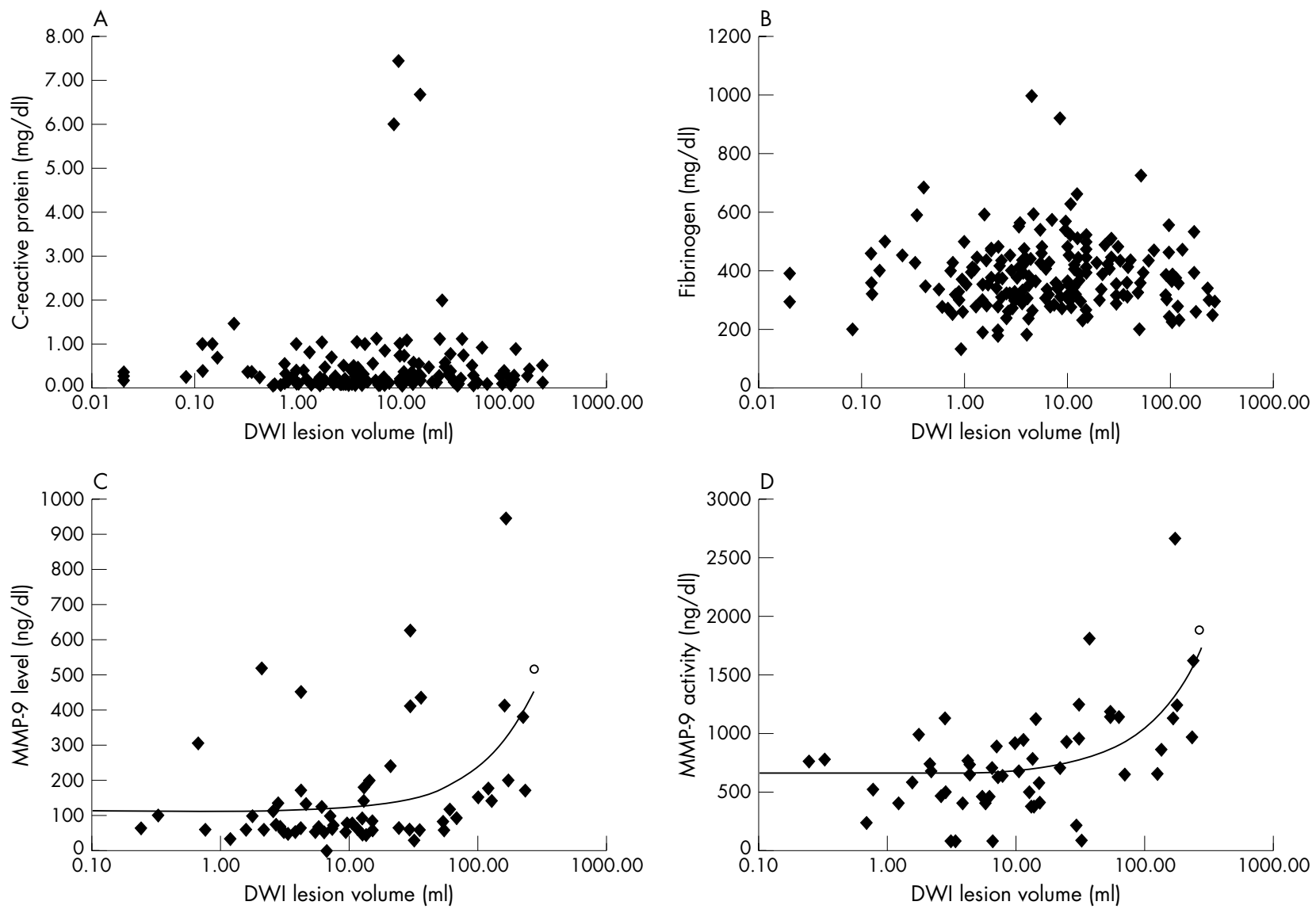

Figure 2 DWI volume and novel risk factors. (A) CRP. (B) Fibrinogen. (C) MMP-9 level. (D) MMP-9 activity. Linear correlation of DWI volume and MMP-9 level (O) or MMP-9 activity (๑).

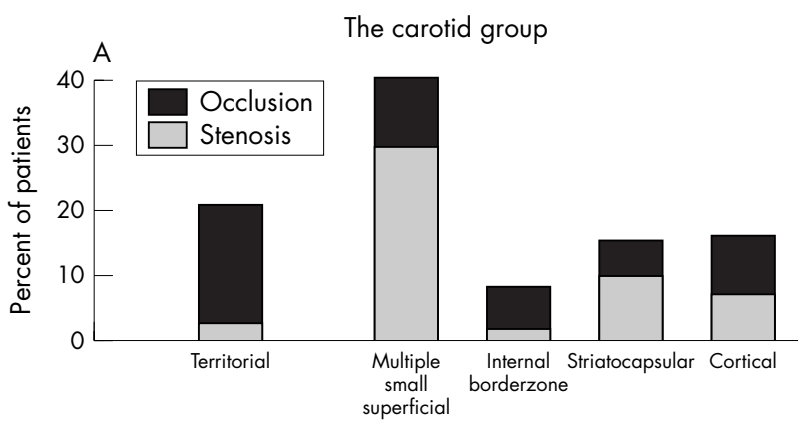

DWI pattern

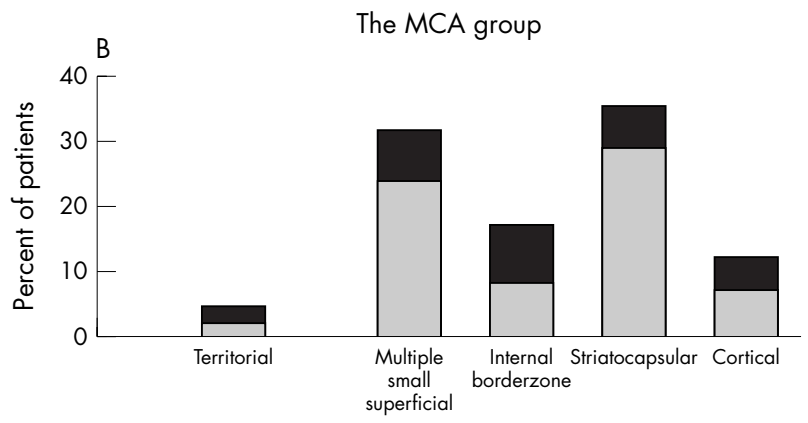

DWI pattern

Figure 3 DWI patterns depending on the degree and site of occlusive lesions. unstable plaque morphology. In the present study, the levels of CRP and fibrinogen were higher in carotid-type patients than in MCA-type patients. Fibrinogen is involved in thrombus formation and is a marker for inflammation. Our results suggest that increased plasma levels of CRP and fibrinogen are not caused by infarction, because the levels of these substances were not related to the size of infarcts. The highest levels of inflammatory markers were observed in our patients who were suspected to have ruptured plaque (total occlusion) or widespread atherosclerosis (irrelevant as well as relevant sites) on the extracranial vessels. MMP-9 appears to play a role in the process of atherosclerosis, particularly in the rupturing of plaques. ${ }^{20}$ It was reported recently that the concentration, production, and expression of MMP-9 were significantly higher in patients with unstable carotid plaques. ${ }^{20}$ However, in the present study, MMP-9 levels and activities were correlated with infarct size rather than with the site of atherosclerosis.

\section{DWI lesion patterns of carotid and MCA}

\section{atherosclerosis are distinct}

Several reports have been based on DWI patterns in patients with carotid or MCA atherosclerotic disease. However, direct comparisons between the DWI patterns of carotid and MCA atherosclerosis have seldom been reported. Our data indicate that carotid and MCA atherosclerosis have distinct patterns of clinical and radiological features. Although a variety of different mechanisms that can cause stroke were suspected in patients with MCA atherosclerosis in the present study, territorial infarcts that would indicate rupturing of a plaque were rarely observed; rather, internal borderzone infarcts that 
are indicative of haemodynamic stroke were the DWI pattern that was observed most frequently in patients with occlusion of the MCA. By contrast, territorial infarcts were the most frequent DWI pattern and observed in $21 \%$ of the carotidtype patients in the present study, which compares favourably with the frequency (29.4\%) of such infarcts in a previous study of patients with carotid disease. ${ }^{21}$

Why did patients with MCA atherosclerosis in the present study exhibit a low frequency of territorial infarcts according to the DWI data? There are several possible explanations. First, plaque disruption may be minor and thrombus formation may be minimal in patients with MCA atherosclerosis, thereby resulting in the formation of thrombi that are smaller than those that result from carotid atherosclerosis. Although there are numerous reports of microembolic signals in patients with MCA atherosclerosis, there has seldom been any pathological confirmation of gross thrombosis in such patients. ${ }^{14}$ In the same manner, the higher level of inflammatory markers (CRP and fibrinogen) in carotidtype patients than in MCA-type patients in this study may account for a high frequency of territory infarcts in carotidtype patients. It was reported that the degree of plaque disruption determines the nature of the ensuing clinical state, $^{22}{ }^{23}$ and rupture of unstable plaque and subsequent formation of a macrothrombus are associated with persistent occlusion at the site of disruption resulting in territory infarcts on DWI. ${ }^{24}$

Second, haemodynamic insufficiency may be the primary cause of stroke in patients with MCA atherosclerosis. Recently, Sliwka et al failed to detect microembolic signals in patients with chronic MCA stenosis (either symptomatic or asymptomatic) and suggested that haemodynamic insufficiency, rather than microembolism, caused recurrent ischemic events in those patients. ${ }^{25}$ The high prevalence of internal borderzone infarcts in patients with MCA atherosclerosis in the present study suggests that haemodynamic insufficiency might be an important cause of stroke in patients with MCA atherosclerosis. ${ }^{12}{ }^{14}$

\section{Differential therapeutic approach may be needed}

One of the main concerns in the treatment and prevention of ischemic stroke is the stabilisation of plaques. The use of statin after ischemic stroke is associated with a reduction in CRP levels and an improved prognosis that is independent of lowering lipid levels; the greatest reduction of risk is observed in patients with relatively high CRP levels ${ }^{26}$ Our examination of novel risk factors and DWI patterns indicated that MCA atherosclerosis is associated with plaques that are relatively more stable than those observed in patients with carotid atherosclerosis; patients with MCA atherosclerosis had lower levels of CRP and a higher frequency of haemodynamic strokes than patients with carotid atherosclerosis. Therefore, it is unlikely that patients with MCA atherosclerosis would benefit greatly from treatment with statin. Further studies are required to elucidate the effects of plaque stabilisers such as statin in patients with large intracranial stenosis.

\section{Limitations and conclusions}

There are several limitations to the present study that deserve mention. First, plaque rupture could not be confirmed pathologically. Thromboembolism does not necessarily mean that plaque rupture caused the emboli. The emboli could easily be explained by a clot superimposed upon moderate to severe arterial narrowing with subsequent breaking loose of the clot and intracranial embolisation. However, our present results corroborate findings of previous clinical and pathological studies of carotid and MCA atherosclerosis. ${ }^{1422-24}$ Moreover, it is unlikely that breaking loose of the clot rather than rupture of plaque produces macroemboli resulting in territorial infarcts on DWI. In addition, it is possible that small cortical infarcts (even internal borderzone infarcts) are caused by small thrombi from minor plaque ruptures. Further studies using sonographic monitoring are needed to detect microembolic signals. ${ }^{13}$ Second, unfortunately, not every patients underwent vascular study within the first few hours following stroke. Therefore, an unknown number of cases might have been missed because recanalisation usually occurs after stroke. Third, improved imaging techniques are available that apparently reveal pathological changes in plaques that are associated with carotid atherosclerosis. ${ }^{27-29}$ These techniques may permit clinicians to distinguish between intra- and extracranial plaques, but they were not used in the present study. Finally, the levels and activities of MMP-9 were not evaluated in all patients in the present study, particularly those that were enrolled in the early phase of the study.

In summary, our results indicate that novel, rather than conventional, risk factors for the rupture of plaques reveal clinical and radiological differences between patients with carotid and MCA atherosclerosis. In addition, it appears that although the rupture of unstable plaques is frequently the cause of thrombotic carotid occlusion, this occurs rarely (if ever) in intracranial vessels. Therefore, differential therapeutic approaches may be required for the treatment of intraand extracranial atherosclerosis.

\section{Authors' affiliations}

O Y Bang, P H Lee, M A Lee, I S Joo, K Huh, Department of Neurology, School of Medicine, Ajou University, Suwon, Republic of Korea S R Yoon, Department of Neuroradiology, Shin Hospital, Suwon, Republic of Korea

This work was supported by a Korean Research Foundation Grant (KRF2003-041-E00228) to OYB.

Competing interests: none declared

\section{REFERENCES}

1 Lammie GA, Sandercock PA, Dennis MS. Recently occluded intracranial and extracranial carotid arteries. Relevance of the unstable atherosclerotic plaque. Stroke 1999;30:1319-25.

2 Masuda J, Ogata J, Yutani C, et al. Artery-to-artery embolism from a thrombus formed in stenotic middle cerebral artery. Report of an autopsy case. Stroke 1987; 18:680-4.

3 Nishida N, Ogata J, Yutani C, et al. Cerebral artery thrombosis as a cause of striatocapsular infarction. Cerebrovasc Dis 2000;10:151-4.

4 Schumacher HC, Tanji K, Mangla S, et al. Histopathological evaluation of middle cerebral artery after percutaneous intracranial transluminal angioplasty. Stroke 2003;34:e170-3.

5 Wong KS, Huang YN, Gao S, et al. Intracranial stenosis in Chinese patients with acute stroke. Neurology 1998;50:812-3.

6 Adams HP Jr, Bendixen BH, Kappelle U, et al. Classification of subtype of acute ischemic stroke. Definitions for use in a multicenter clinical trial. TOAST. Trial of Org 10172 in Acute Stroke Treatment. Stroke 1993;24:35-41.

7 Samuels OB, Joseph GJ, Lynn MJ, et al. A standardized method for measuring intracranial arterial stenosis. AJNR Am J Neuroradiol 2000;21:643-6.

8 North American Symptomatic Carotid Endarterectomy Trial Collaborators. Beneficial effect of carotid endarterectomy in symptomatic patients with highgrade stenosis. N Engl J Med 1991;325:445-53.

9 Bang OY, Lee PH, Joo SY, et al. Frequency and mechanisms of stroke recurrence after cryptogenic stroke. Ann Neurol 2003;54:227-34.

10 Bogousslavsky J, Regli F. Centrum ovale infarcts: subcortical infarction in the superficial territory of the middle cerebral artery. Neurology 1992;42:1992-8

11 Heinsius T, Bogousslavsky J, Van Melle G. Large infarcts in the middle cerebral artery territory. Etiology and outcome patterns. Neurology 1998;50:341-50.

12 Lee PH, Bang OY, Oh SH, et al. Subcortical white matter infarcts. Comparison of superficial perforating artery and internal border-zone infarcts using
diffusion-weighted magnetic resonance imaging. Stroke 2003;34:2630-5.

13 Kimura K, Minematsu K, Koga M, et al. Microembolic signals and diffusionweighted MR imaging abnormalities in acute ischemic stroke. AJNR Am J Neuroradiol, 2001;22, 1037-42.

14 Nakano S, Yokogami K, Ohta H, et al. CT-defined large subcortical infarcts: correlation of location with site of cerebrovascular occlusive disease. AJNR Am J Neuroradiol 1995;16:1581-5.

15 Bogousslavsky J, Barnett $\mathrm{HJ}$, Fox AJ, et al. Atherosclerotic disease of the middle cerebral artery. Stroke 1986;17:1112-20. 
16 Akins PT, Pilgram TK, Cross DT 3rd, et al atural history of stenosis from intracranial atherosclerosis by serial angiography. Stroke 1998;29:433-8.

17 Gorelick PB, Caplan LR, Hier DB, et al. Racial differences in the distribution of anterior circulation occlusive disease. Neurology 1984;34:54-9.

18 Inzitari D, Hachinski VC, Taylor DW, et al. Racial differences in the anterio circulation in cerebrovascular disease. How much can be explained by risk factors? Arch Neurol 1990;47:1080-4.

19 Van Der Meer IM, De Maat MP, Hak AE, et al. C-reactive protein predicts progression of atherosclerosis measured at various sites in the arterial tree: the Rotterdam Study. Stroke 2002;33:2750-5.

20 Loftus IM, Naylor AR, Goodall S, et al. Increased matrix metalloproteinase-9 activity in unstable carotid plaques: a potential role in acute plaque disruption. Stroke 2000;31:40-7.

21 Szabo K, Kern R, Gass A, et al. Acute stroke patterns in patients with internal carotid artery disease: a diffusion-weighted magnetic resonance imaging study. Stroke 2001;32:1323-9.

22 Davies MJ, Thomas AC. Plaque fissuring: the cause of acute myocardial infarction, sudden ischaemic death and crescendo angina. Br Heart J 1985;53:363-73.
23 Hennerici M, Hulsbomer $\mathrm{HB}$, Hefter $\mathrm{H}$, et al. Natural history of asymptomatic extracranial arterial disease. Results of a long-term prospective study. Brain 1987; 110:777-91.

24 Fuster V, Badimon L, Badimon JJ, et al. The pathogenesis of acute coronary artery disease and acute coronary syndromes (1). N Engl J Med 1992;326:242-50

25 Sliwka U, Klotzsch C, Popescu O, et al. Do chronic middle cerebral artery stenoses represent an embolic focus? A multirange transcranial Doppler study. Stroke 1997;28:1324-7.

26 Di Napoli M, Papa F. Inflammation, statins, and outcome after ischemic stroke. Stroke 2001;32:2446-7.

27 Yuan C, Miller ZE, Cai J, et al. Carotid atherosclerotic wall imaging by MRI. Neuroimaging Clin N Am 2002;12:391-401

28 Fayad ZA. Noncoronary and coronary atherothrombotic plaque imaging and monitoring of therapy by MRI. Neuroimaging Clin N Am 2002;12:461-71.

29 Choudhury RP, Fuster V, Badimon JJ, et al. MRI and characterization of atherosclerotic plaque: emerging applications and molecular imaging. Arterioscler Thromb Vasc Biol 2002;22:1065-74.

\section{bmjupdates+}

bmjupdates+ is a unique and free alerting service, designed to keep you up to date with the medical literature that is truly important to your practice.

bmjupdates+ will alert you to important new research and will provide you with the best new evidence concerning important advances in health care, tailored to your medical interests and time demands.

Where does the information come from?

bmjupdates+ applies an expert critical appraisal filter to over 100 top medical journals A panel of over 2000 physicians find the few 'must read' studies for each area of clinical interest

Sign up to receive your tailored email alerts, searching access and more...

www.bmjupdates.com 